\title{
Modelagem e predição da situação acadêmica de alunos de educação a distância.
}

\author{
Douglas Detoni $^{1}$, Ricardo Araujo Matsumura ${ }^{1}$, Cristian Cechinel $^{2}$ \\ ${ }^{1}$ Centro de Desenvolvimento Tecnológico - Universidade Federal de Pelotas (UFPEL) \\ Caixa Postal 1 - 96010-610 - Pelotas - RS - Brasil \\ ${ }^{2}$ Centro de Educação a Distância - Universidade Federal de Pelotas (UFPEL) \\ Caixa Postal 630 - 96010-000 - Pelotas - RS - Brasil \\ \{ddetoni,ricardo\}@inf.ufpel.edu.br, contato@cristiancechinel.pro.br
}

\begin{abstract}
The possibility of predicting the risk of a student failing a course is useful for tutors and teachers, as this allows them to change their methods to avoid failures. In this paper we show results of attempts to train machine learning models to perform this task, using only interaction counts as attributes. We show that Bayesian networks are adequate for the problem and that introducing attributes derived from the counts (e.g. means) are useful for more accurate predictions when data is sparse and that using data sets balanced in relation to approved and disapproved classes increases the prediction of disapproved. We also show the possibility of training models in different source of examples, such as between different groups and semesters.
\end{abstract}

Resumo. A possibilidade de prever com antecedência o risco de reprovação de um estudante em um curso a distância é de grande valia para professores e tutores. Neste trabalho mostramos resultados da aplicação de técnicas de aprendizado de máquina nesta tarefa, utilizando como atributos unicamente contagens de interações ao longo do tempo. Mostramos que redes bayesianas são adequadas ao problema. Vimos que a introdução de atributos derivados das contagens (e.g. médias) são úteis para previsões mais precisas quando a quantidade de dados é esparsa e que utilizando conjuntos de dados balanceados em relação a aprovados e reprovados aumenta a predição dos reprovados. Mostramos ainda a possibilidade de treinar os modelos em diferentes situações, como entre turmas e entre semestres diferentes.

\section{Introdução}

A Educação a Distância (EAD) no Brasil tem se consolidado, com diversos estudantes optando por essa modalidade de ensino para ampliar suas formações e realização profissional. Em 2012, segundo dados Censo EAD [CensoEaD.BR 2013], foram ofertados 9.376 cursos. Um total de 5.772.466 matrículas foram registradas, 52,5\% de aumento em relação a 2011. Já o número de conclusões, em 2012, foi de 1.589.374. Esses dados mostram a evolução da Educação a Distância e como ela está se tornando uma ferramenta muito importante na formação dos cidadãos brasileiros. Contudo, a EAD ainda enfrenta alguns obstáculos a serem ultrapassados. Resistência de educandos e educadores, desafios organizacionais e custos de produção são alguns deles mas, sem dúvida, um dos maiores 
obstáculos é a evasão de alunos dos cursos e instituições. No ano de 2012, a evasão foi de $11,74 \%$ nos cursos autorizados, segundo dados do Censo EAD.

Um dos principais diferenciais de cursos de EAD é a grande quantidade de dados gerada pelas interações no ambiente educacional, o que abre novas possibilidades para estudar e compreender estas interações [Hamalainen and Vinni 2011]. Assim, algumas áreas de pesquisas surgiram nos últimos anos com intuito de auxiliar em questões como essas. A Educational Data Mining (EDM) é uma área de pesquisa interdisciplinar que lida com o desenvolvimento de métodos para explorar dados originados no contexto educacional [Romero and Ventura 2010]. Juntamente com a EDM temos a Learning Analytics (LA), outra área de pesquisa emergente. Segundo definição criada na conferência Learning Analytics and Knowledge (LAK) em 2012, LA busca medir, coletar, analisar e reportar dados sobre estudantes e seus contextos, com o propósito de entender e otimizar seu aprendizado e o ambiente onde ele ocorre. A interação dos alunos com os ambientes virtuais de aprendizagem (AVA) proveem os dados que alimentam as pesquisas na área e possibilitam a descoberta de novos conhecimentos.

Um dos grandes desafios dos pesquisadores é desenvolver métodos capazes de prever o comportamento dos estudantes, de modo a possibilitar a intervenção de professores/tutores, ou demais envolvidos, visando resgatar o estudante antes que ele reprove [Macfadyen and Dawson 2010]. Outros objetivos são detectar estudantes que precisam de ajuda, prever o desempenho, classificar diferentes grupos de estudantes, detectar trapaças no sistema, entre outros [Romero and Ventura 2010]. Segundo Romero and Ventura (2010), existe a necessidade de desenvolver ferramentas específicas e fáceis de usar, para que professores/tutores não familiarizados com as técnicas de EDM e LA também possam se valer das descobertas das áreas. É importante também integrar as ferramentas a AVAs tradicionalmente já usados. Outro aspecto a ser trabalhado é a padronização dos dados e modelos. Muitas ferramentas possuem padrões próprios e específicos, os quais não podem ser integrados por outras ferramentas.

O principal objetivo deste trabalho é desenvolver e testar técnicas e modelos de aprendizado de máquina na predição da reprovação de alunos de educação a distância utilizando o registro de interações entre alunos, tutores e professores em cursos da Universidade Federal de Pelotas no ambiente Moodle.

Adicionalmente, é nosso objetivo analisar unicamente atributos agregados - i.e. números totais de interações, sem especificar o tipo de atividade sendo realizada pelo estudante. Este objetivo é motivado pela heterogeneidade de atividades entre cursos e plataformas; ao utilizar como atributo atividades específicas (e.g. fórum, chat), acaba-se por ancorar a técnica aos cursos específicos sendo analisados, tornando-a pouco generalizável.

Este trabalho está estruturado da seguinte maneira. A seção 2 descreve alguns trabalhos relacionados com a predição da situação de acadêmicos em AVAs que se aproximam do presente trabalho. A seção 3 apresenta o contexto de coleta dos dados e o pré-processamento realizado. Na seção 4 são descritos as análises realizadas e na seção 5 os resultados encontrados. As considerações finais e propostas de trabalhos futuros são apresentadas na seção 6. 


\section{Trabalhos Relacionados}

A previsão do desempenho, ou situação, acadêmica de alunos é um tema muito estudado entre pesquisadores. Manhaes, da Cruz, Costa, Zavaleta, and Zimbrao (2011) utilizaram técnicas de mineração de dados para prever a evasão de estudantes em cursos presenciais da Escola Politécnica da Universidade do Rio de Janeiro. Dez modelos diferentes foram testados, com acurácia média variando entre $75 \%$ e $80 \%$, com Perceptron de Múltiplas Camadas e Florestas Aleatórias apresentando os melhores desempenhos.

Gottardo, Kaester, and Noronha (2012), apresentam resultados de tentativas de prever a avaliação de desempenho de alunos de educação a distância. Os autores relatam taxas de precisão acima de $74 \%$, com o uso de uma grande variedade de atributos. O presente trabalho diferencia-se ao tentar prever a reprovação de alunos e não seus desempenhos específicos, além de prover uma análise mais detalhada utilizando unicamente atividades agregadas.

Rodrigues, de Medeiros, and Gomes (2013) apresentaram um estudo da viabilidade do uso de um modelo de regressão linear para também prever o desempenho dos alunos. Este trabalho também utilizou dados do ambiente virtual de aprendizagem Moodle, além da utilização de séries temporais. Diversos atributos foram utilizados, incluindo a quantidade de interações ao longo das semanas em fóruns, vídeos, materiais de apoio, entre outros.

Gotardo, Cereda, and Hruschka Junior (2013) realizam a tarefa de predição do desempenho do aluno utilizando Sistemas de Recomendação e Acoplamento de Classificadores. Os dados são relativos a um curso com 252 alunos. Os dados para treinamento dos classificadores foram extraídos da tabela de logs e separados por tipos, como por exemplo: assignment, forums, upload, entre outros. Cada tipo transformou-se em um atributo, juntamente com a situação acadêmica do aluno. Os classificadores J48 e Naive Bayes foram utilizados no trabalho. O melhor resultado foi aproximadamente $80 \%$ de predição, utilizando $40 \%$ do conjunto de dados, com o classificador Naive Bayes.

\section{Descrição dos Dados}

\subsection{Contexto dos cursos}

A Universidade Federal de Pelotas (UFPEL) oferece cursos de graduação, pós-graduação e extensão na modalidade a distância. São mais de 4000 alunos matriculados e espalhados por diversos polos do Rio Grande do Sul.

Para a realização do trabalho, foram obtidos os dados anonimizados dos cursos a distância de Licenciatura Educação do Campo (CLEC) e de Licenciatura em Pedagogia (CLPD). É importante notar que estes cursos seguem um modelo de execução das disciplinas que difere do modelo presencial convencional. Normalmente, um curso é dividido em semestres, onde diversas disciplinas são ministradas paralelamente ao longo do mesmo. No caso do CLEC e do CLPD, as disciplinas são executadas de maneira sequencial (com no máximo 2 disciplinas acontecendo em paralelo). Normalmente são executadas em torno de 5 disciplinas por semestre com duração média de cinco a sete semanas.

Foram utilizadas disciplinas do primeiro e segundo semestre dos cursos citados. Ambos os semestres ocorreram no ano de 2013, totalizando 329 estudantes únicos. Con- 
tabilizando os 4 semestres utilizados, temos um total de 604 alunos, 72 tutores e 80 professores. A Tabela 1 apresenta o número de alunos para cada situação.

Tabela 1. Total de alunos em cada curso e semestre

\begin{tabular}{|c|c|c|c|c|}
\hline Curso & Semestre & Alunos & Aprovados & Reprovados \\
\hline \multirow{2}{*}{ Educação do Campo (CLEC) } & $1^{\mathrm{o}}$ & 133 & 103 & 30 \\
\cline { 2 - 5 } & $2^{\mathrm{o}}$ & 94 & 88 & 6 \\
\hline \multirow{2}{*}{ Pedagogia (CLPD) } & $1^{\mathrm{o}}$ & 196 & 137 & 59 \\
\cline { 2 - 5 } & $2^{\mathrm{o}}$ & 172 & 119 & 53 \\
\hline
\end{tabular}

\subsection{Coleta e Pré-processamento dos Dados}

Os dados foram extraídos dos registros de acesso dos cursos fornecidos pela plataforma Moodle. Destes registros, para cada interação de cada usuário, extraiu-se apenas o identificador do usuário, sua função (professor, tutor ou aluno) e a data e hora da interação. Assim, ao contrário de trabalhos anteriores, procuramos utilizar apenas o número total de interações dos estudantes, tutores e professores com o sistema, sem detalhamento do tipo de atividade sendo realizada. Isto permite que os classificadores gerados sejam aplicáveis a diferentes cursos, plataformas e execuções, sem gerar uma dependência em atributos excessivamente específicos. No total foram extraídas cerca de 835 mil interações.

Cada aluno foi rotulado como aprovado ou reprovado em cada disciplina de acordo com a situação informada nos registros acadêmicos. Cada disciplina possui duração de 7 semanas e as interações de cada aluno foram agrupadas de forma semanal. Assim, cada aluno em uma disciplina é descrito por um vetor de 7 valores indicando o número total de interações daquele aluno em cada semana.

Considerando que o objetivo principal é predizer a situação final do aluno com a maior antecedência possível dentro da disciplina, foram gerados diversos conjuntos de dados com base nas combinações incrementais das sete semanas de interações existentes para a disciplina. Assim, foram gerados conjuntos de dados contendo somente a primeira semana de interações, contendo a primeira e a segunda semana, e assim por diante, até ser gerado o conjunto de dados com todas as 7 semanas de interações. Um conjunto de dados consiste em todos os exemplos de alunos de um dado semestre de um curso.

\subsection{Atributos Derivados}

Ainda que a série temporal gerada pelo número absoluto de interações dos alunos possa ser útil, geramos também um conjunto de atributos derivados que podem auxiliar na contextualização desses valores. O conjunto total de atributos utilizados e a descrição dos mesmos são apresentados na Tabela 2. Todos atributos são derivados unicamente na contagem total de interações e relações entre interações de alunos, tutores e professores. Este conjunto de atributos foi escolhido de modo que certos aspectos dos dados originais fossem enfatizados. Esse é o caso da média, mediana e número de semanas com zero interações. Os demais foram criados com base em hipóteses, tentando revelar características ainda não conhecidas ou relações entre os dados de professores e tutores com os alunos. A principal hipótese é de que o número absoluto de interações dos estudantes tem pouco valor, sendo necessário colocá-lo em contexto do número de interações da turma como um todo e dos tutores e professores. Também foi buscada uma inter-relação entre os 
dados de um exemplo de aluno com os dados dos demais exemplos de aluno da disciplina, caso este do fator de empenho.

Tabela 2. Conjunto de atributos.

\begin{tabular}{|c|c|}
\hline Atributo & Descrição \\
\hline Identificador do aluno & Número para a identificação do aluno \\
\hline Situação Acadêmica & Situação final do aluno na disciplina. Ex.: Aprovado ou Reprovado \\
\hline Interações por Semana (1 até 7 semanas) & Número de interações na semana. E.: Sem1 $=80$, Sem2 $=50$. \\
\hline Média & Média do total de interações pelo número de semanas. \\
\hline Mediana & Mediana do conjunto de interações por semana. \\
\hline Semanas Zeradas & Número de semanas com zero interações \\
\hline Média da Diferença & Média da diferença entre a semana $s_{i}$ e $s_{i+1}$. \\
\hline Razão com Professores & Razão entre o total de interações do aluno e dos professores. \\
\hline Razão com Tutores & Razão entre o total de interações do aluno e dos tutores. \\
\hline Fator de Empenho (1 até 7 semanas) & Razão entre as interações da semana do aluno e a média da turma na mesma semana. \\
\hline
\end{tabular}

\subsection{Balanceamento do conjunto}

De modo a tentar amenizar a disparidade entre aprovados e reprovados nos conjuntos de exemplos, foi proposta uma análise utilizando o conjunto de exemplos balanceado. Kotsiantis (2009) fez um levantamento de alguns trabalhos sobre conjunto de dados desbalanceados. Japkowicz and Stephen (2002) discutem o efeito do desbalanceamento em conjuntos de dados. Dois métodos para gerar novos exemplos são considerados. Geração randomica com base em toda a classe minoritária e replicação de exemplos raros, ou seja, exceções no conjunto. Neste trabalho utilizou-se uma maneira mais simplista de balanceamento. Foi usado um conjunto de exemplos com atributos derivados como base, onde os exemplos de alunos reprovados foram sendo replicados até que o número de exemplos fosse igualado. Ao serem replicados, uma pequena mutação foi feita em uma das posições do vetor de interações por semana, de modo a balancear o conjunto não com exemplos exatamente iguais, mas sim com pequenas variações dos existentes.

\section{Metodologia de Análise}

Neste artigo, estamos interessados em verificar a eficácia de diferentes modelos em prever a situação de alunos em diferentes situações. Em particular, focamos em três casos: a) utilizando somente o número absoluto de interações dos estudantes, b) adicionando os atributos derivados e c) balanceando o conjunto de exemplos com atributos derivados. $\mathrm{O}$ primeiro caso visa identificar o potencial do modelo de dados constituído somente pela situação do aluno e as interações que ele teve em cada semana. O segundo caso utiliza as mesmas informações do primeiro, porém acrescentando os atributos derivados (média, mediana, média da diferença, semanas zeradas, razão com os professores, razão com os tutores e o fator de empenho de cada semana). O terceiro caso visa analisar o desempenho dos modelos quando treinados com conjunto de exemplos balanceado.

Para cada análise, dividiu-se os conjuntos de exemplos em um conjunto de treinamento e um conjunto de teste. Esta divisão foi feita de duas formas para testar duas situações distintas. Na primeira forma, denominada "entre turmas", a divisão é feita dentro de um mesmo semestre do mesmo curso, utilizando turmas distintas para treino e teste. Na segunda forma, denominada "entre semestres", a divisão é feita entre semestres distintos. 
Para tanto, cada conjunto de dados foi dividido aleatoriamente em dois conjuntos contendo a mesma quantidade de alunos, ou seja, cada turma de alunos foi transformada em duas turmas (A e B). No primeiro formato (entre turmas) uma turma (ex: turma A) de um semestre de um curso foi utilizada para treinar o modelo, e a outra turma (ex: turma B) daquele semestre daquele curso foi utilizada para avaliar o modelo. No segundo formato (entre semestres), cada uma das turmas (A e B) de cada semestre foi utilizada para treinar os modelos, e os mesmos foram avaliados com as turmas A e B do semestre seguinte (e vice-versa).

Esse processo de divisão foi repetido 5 vezes, gerando conjuntos aleatórios diferentes a cada repetição. As médias dos resultados da aplicação dos classificadores nestas repetições são reportadas.

Os seguintes modelos foram gerados e avaliados utilizando a ferramenta WEKA: Rede Bayesiana, Rede Neural (Perceptron de Múltiplas Camadas), J48 e Floresta Aleatória.

\section{Resultados}

De modo geral, as acurácias médias de classificação alcançaram altos índices já para os modelos que utilizavam somente os dados das interações da primeira semana, tendo sido possível, por exemplo, gerar modelos com acurácia média superior a 90\% para os casos a) e b).

No entanto, medidas de acurácia dos classificadores não são adequadas nesses casos, já que as classes não são balanceadas - há muito mais candidatos retidos do que evadidos, fazendo com que classificadores que somente indicam retenção tenham já alta acurácia sem serem realmente úteis. Assim, focamos aqui na capacidade dos classificadores em indicar corretamente reprovações - isto é, a fração corretamente classificada de estudantes reprovados. No caso c), onde os conjuntos são balanceados, iremos focar na acurácia média.

A precisão na classificação de alunos aprovados manteve-se sempre alta, entre $75 \%$ e $95 \%$, demonstrando que a tarefa de prever reprovação é substancialmente mais difícil.

Por restrições de espaço descreveremos somente os experimentos relacionados ao Curso de Licenciatura em Educação do Campo nos casos a) e b), mas resultados semelhantes foram obtidos para o curso de Pedagogia. Em relação ao caso c), serão apresentados resultados de média onde estão inclusos experimentos de ambos cursos.

\subsection{Classificação utilizando somente número de interações}

Como é possível observar na Tabela 3, os modelos se tornam mais precisos conforme mais semanas são disponibilizadas ao modelo. Quando utilizamos somente as quantidades de interações da primeira semana, as precisões para classificar alunos reprovados são próximas a zero.

À medida que dados de mais semanas são adicionados, os modelos tornam-se, como esperado, mais precisos. Observa-se, no entanto que há uma diminuição de ganhos com o passar das semanas - além da quinta semana novos dados acrescentam muito pouco ao modelo. 
De forma geral, as Redes Bayesianas obtiveram os melhores resultados, com exceção da primeira e segunda semanas, onde as Florestas Aleatórias obtiveram melhores resultados.

Tabela 3. Precisões para classificação de reprovados - experimento sem utilização de atributos

\begin{tabular}{|c|c|c|c|c|c|c|c|c|}
\hline \multirow{3}{*}{ Caso } & \multirow{2}{*}{ Modelo } & \multicolumn{7}{|c|}{ Semana } \\
\cline { 3 - 9 } & & S1 & S2 & S3 & S4 & S5 & S6 & S7 \\
\hline \multirow{4}{*}{ Entre Semestres } & Rede Bayesiana & 0 & 0,22 & 0,44 & 0,51 & 0,6 & 0,63 & $\mathbf{0 , 6 4}$ \\
\cline { 2 - 9 } & Rede Neural & 0 & 0,16 & 0,33 & 0,45 & 0,49 & 0,51 & 0,51 \\
\cline { 2 - 9 } & J48 & 0 & 0,21 & 0,37 & 0,4 & 0,52 & 0,56 & 0,56 \\
\cline { 2 - 9 } Entre Turmas & Floresta Aleatória & 0,1 & 0,33 & 0,36 & 0,4 & 0,48 & 0,51 & 0,54 \\
\hline & Rede Bayesiana & 0 & 0,24 & 0,45 & 0,51 & 0,58 & 0,62 & $\mathbf{0 , 6 6}$ \\
\cline { 2 - 9 } & Rede Neural & 0 & 0,14 & 0,33 & 0,46 & 0,49 & 0,53 & 0,56 \\
\cline { 2 - 9 } & J48 & 0 & 0,2 & 0,39 & 0,42 & 0,51 & 0,59 & 0,6 \\
\cline { 2 - 9 } & Floresta Aleatória & 0,1 & 0,28 & 0,33 & 0,41 & 0,5 & 0,56 & 0,61 \\
\hline
\end{tabular}

\subsection{Classificação utilizando atributos derivados}

Para os modelos treinados com atributos derivados temos um comportamento semelhante aos do experimento anterior. De modo geral os modelos também tornam-se mais precisos a medida que as informações de interação de cada semana vão sendo disponibilizadas e Redes Bayesianas se mostraram o modelo mais adequado novamente. Observando apenas a última semana, não há ganhos na precisão da classificação.

Tabela 4. Precisões para classificação de reprovados - experimento com a utilização de atributos

\begin{tabular}{|c|c|c|c|c|c|c|c|c|}
\hline \multirow{2}{*}{ Testes } & \multirow{2}{*}{ Modelo } & \multicolumn{8}{|c|}{ Semana } \\
\cline { 3 - 9 } & & S1 & S2 & S3 & S4 & S5 & S6 & S7 \\
\hline \multirow{4}{*}{ Entre Semestres } & Rede Bayesiana & 0,32 & 0,48 & 0,54 & 0,54 & 0,65 & 0,66 & $\mathbf{0 , 6 3}$ \\
\cline { 2 - 9 } & Rede Neural & 0,07 & 0,22 & 0,38 & 0,42 & 0,46 & 0,55 & 0,58 \\
\cline { 2 - 9 } & J48 & 0,02 & 0,25 & 0,34 & 0,37 & 0,48 & 0,5 & 0,53 \\
\cline { 2 - 9 } & Floresta Aleatória & 0,06 & 0,26 & 0,3 & 0,37 & 0,41 & 0,47 & 0,46 \\
\hline \multirow{5}{*}{ Entre Turmas } & Rede Bayesiana & 0,35 & 0,48 & 0,52 & 0,57 & 0,64 & 0,68 & $\mathbf{0 , 6 7}$ \\
\cline { 2 - 9 } & Rede Neural & 0,08 & 0,24 & 0,41 & 0,44 & 0,49 & 0,57 & 0,59 \\
\cline { 2 - 9 } & J48 & 0,05 & 0,27 & 0,36 & 0,39 & 0,49 & 0,57 & 0,64 \\
\cline { 2 - 9 } & Floresta Aleatória & 0,11 & 0,27 & 0,33 & 0,41 & 0,45 & 0,54 & 0,51 \\
\hline
\end{tabular}

\subsection{Classificação utilizando balanceamento e atributos derivados}

Os modelos treinados com conjuto de exemplos balanceado demonstraram um considerável aumento na predição da classe Reprovados, como pode-se ver na Tabela 5. No caso entre turmas, o modelo Rede Bayesiana chegou a $80 \%$ de acurácia média com conjuntos de exemplos variando de 1 a 7 semanas. 
Tabela 5. Média por classificador para modelos gerados entre turmas e com conjunto de exemplos balanceado.

\begin{tabular}{|c|c|c|c|c|c|}
\hline \multirow{2}{*}{ Modelo } & \multirow{2}{*}{ Acurácia \% } & \multicolumn{2}{|c|}{ Aprovados } & \multicolumn{2}{c|}{ Reprovados } \\
\cline { 3 - 6 } & & TP-Rate & FP-Rate & TP-Rate & FP-Rate \\
\hline Rede Bayesiana & $\mathbf{8 0 , 1 8}$ & 0,87 & 0,27 & 0,73 & 0,13 \\
\hline Rede Neural & 79,26 & 0,82 & 0,24 & $\mathbf{0 , 7 6}$ & 0,18 \\
\hline J48 & 77,67 & 0,85 & 0,29 & 0,71 & 0,15 \\
\hline Floresta Aleatória & 78,09 & $\mathbf{0 , 8 8}$ & 0,32 & 0,68 & 0,12 \\
\hline
\end{tabular}

É importante lembra, que neste caso a acurácia é uma medida relevante pois os modelos treinados e testados com conjuntos de exemplos balanceados. Na Tabela 6 temse os resultados do caso "entre semestres", onde é possível observar que os resultados apresentados são bastante parecidos com o caso "entre turmas". Em ambos casos Redes Bayesianas obtiveram os melhores resultados.

Tabela 6. Média por classificador para modelos gerados entre semestres e com conjunto de exemplos balanceado.

\begin{tabular}{|c|c|c|c|c|c|}
\hline \multirow{2}{*}{ Modelo } & \multirow{2}{*}{ Acurácia \% } & \multicolumn{2}{|c|}{ Aprovados } & \multicolumn{2}{c|}{ Reprovados } \\
\cline { 3 - 6 } & & TP-Rate & FP-Rate & TP-Rate & FP-Rate \\
\hline Rede Bayesiana & $\mathbf{7 9 , 6 7}$ & 0,87 & 0,28 & 0,73 & 0,13 \\
\hline Rede Neural & 77,25 & 0,82 & 0,27 & $\mathbf{0 , 7 3}$ & 0,18 \\
\hline J48 & 74,84 & 0,83 & 0,33 & 0,67 & 0,17 \\
\hline Floresta Aleatória & 75,86 & $\mathbf{0 , 8 7}$ & 0,36 & 0,64 & 0,13 \\
\hline
\end{tabular}

\subsection{Comparação dos resultados}

A Figura 1 compara o desempenho obtido por semana utilizando Redes Bayesianas e as duas combinações de atributos. Observa-se que, para as semanas finais, as precisões dos modelos são virtualmente idênticas. No entanto, para semanas iniciais, a utilização dos atributos derivados torna-se substancialmente vantajosa. A partir da quarta semana os resultados se aproximam, convergindo para essencialmente a mesma precisão na última semana.

Figura 1. Comparação de desempenho a cada semana utilizando Redes Bayesianas e diferentes combinações de atributos

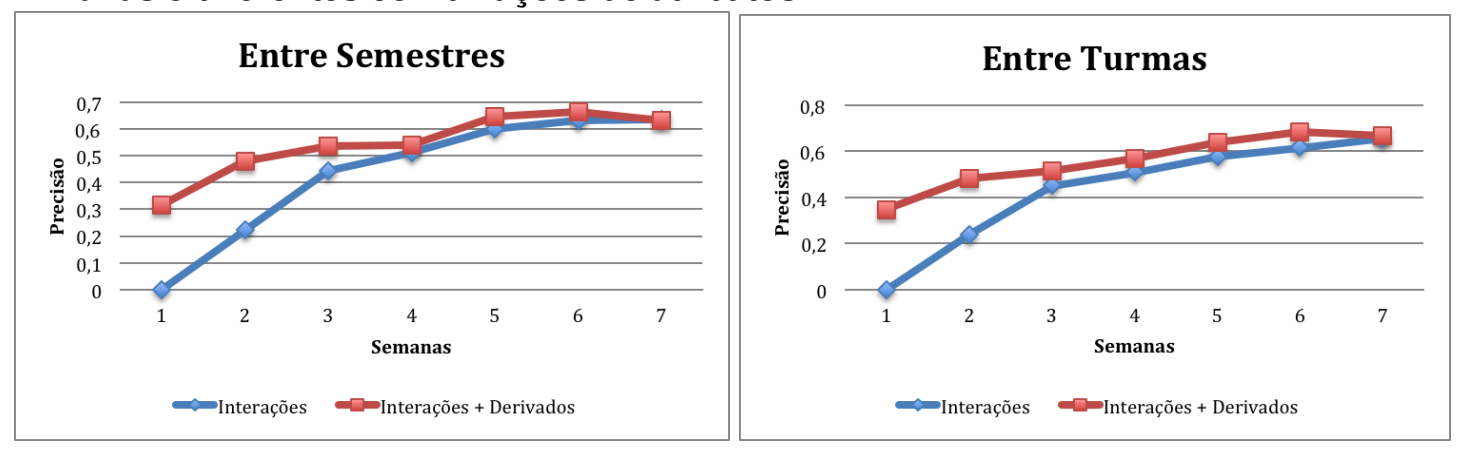


Observa-se ainda que a precisão a cada semana é essencialmente a mesma independente se o modelo foi treinado entre turmas ou entre semestres (Figura 2). Este é um resultado interessante, pois demonstra uma invariância no resultado em relação a origem do treinamento. Isto é, um modelo pode ser treinado com dados de um semestre para ser aplicado no semestre posterior, bem como treinado em um conjunto de estudantes para ser aplicado em outros estudantes.

Figura 2. Comparação de precisão por semana utilizando Rede Bayesiana e todos atributos disponíveis

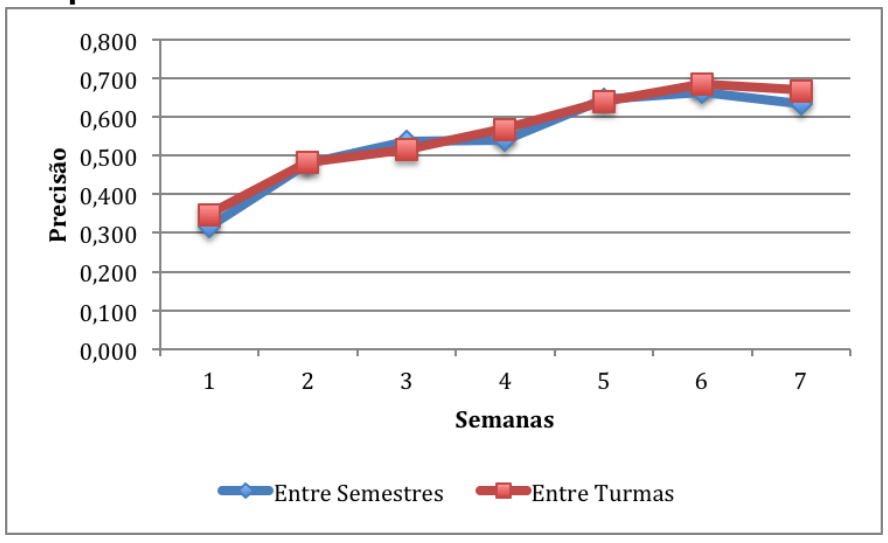

Os modelos treinados e testados com conjuntos de exemplos balanceados não serão comparados com os casos acima, pois entende-se que utilizar conjuntos de exemplos balanceados ainda carece de maiores pesquisas, de modo a validar o método. Contudo, pode-se comparar os casos balanceados "entre turmas" e "entre semestres". Como já foi dito, os resultados entre os dois foram muito parecidos, mostrando mais uma vez a possibilidade de se treinar um modelo com dados de um semestre e aplicá-lo no semestre posterior. De modo geral, o que pode ser observado foi uma queda na acurácia média, no caso "entre semestres", de 1,9\%.

\section{Considerações Finais e Trabalhos Futuros}

A possibilidade de prever com antecedência se um estudante de educação a distância corre o risco de não concluir uma disciplina ou curso é de grande valia para professores e tutores, que podem ajustar seus instrumentos pedagógicos para evitar que estes estudantes reprovem ou evadam.

Neste artigo mostramos resultados da aplicação de técnicas de aprendizado de máquina na predição de reprovação de estudantes. Demonstramos que utilizar apenas a quantidade de interações dos alunos é viável para gerar predições razoavelmente precisas, ainda que menos precisas do que trabalhos anteriores que utilizam atributos muito mais específicos; estes, porém, sacrificam generalidade na aplicação em outros contextos, enquanto nossa abordagem é aplicável virtualmente em qualquer situação onde é possível contar interações de qualquer tipo.

Mostramos ainda que utilizar apenas o número de interações dos estudantes leva a uma baixa capacidade preditiva quando poucas semanas de dados estão disponíveis. Derivando-se atributos que levam em conta a quantidade de interações de professores e 
tutores, bem como outros atributos comparativos, e utilizando estes atributos nos modelos, obtém-se um desempenho bastante superior para semanas iniciais ao utilizar-se um modelo capaz de aproveitar estar informações. Observamos que estas derivações não violam nosso requisito de apenas utilizar contagens de interações genéricas, já que ainda não especificamos em nenhum momento que interações estão sendo realizadas.

Por fim, mostramos que redes bayesianas se mostraram o modelo mais adequado para o problema e que é possível treinar o modelo utilizando diferentes fontes de exemplos. Em particular, obtivemos precisões idênticas ao treinar tanto entre turmas, onde o treino é realizado sobre um conjunto de estudantes e a aplicação em outro conjunto disjunto, como entre semestres, onde o treino ocorre utilizando estudantes de um semestre e aplica-se em outro semestre.

Trabalhos futuros incluem reduzir a diferença de desempenho utilizando-se apenas contagem de interações e utilizando-se atributos mais específicos. Para isso, parece ser necessário derivar outros atributos ou encontrar melhores modelos capazes de fazer uso destes atributos. Adicionalmente, é necessário ampliar os testes aqui realizados para outros cursos, inclusive em outras instituições, de forma a melhor validar o modelo.

\section{Referências}

CensoEaD.BR (2013). http://www.abed.org.br/censoead/censoEAD . BR_2012_pt.pdf.

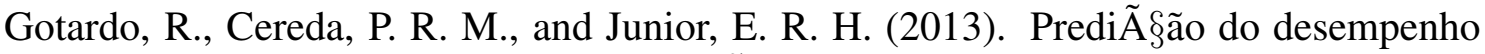
do aluno usando sistemas de recomenda $\tilde{A} \oint \tilde{a} o$ e acoplamento de classificadores. XXIV

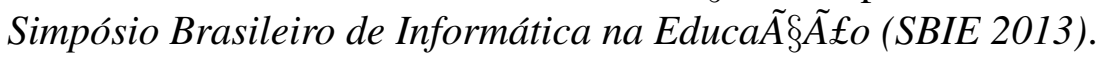

Gottardo, E., Kaester, C., and Noronha, R. V. (2012). Previsão de desempenho de estudantes em cursos ead utilizando mineração de dados: uma estratégia baseada em séries temporais. Anais do XXIII SBIE.

Hamalainen, W. and Vinni, M. (2011). Classifiers for educational data mining. Chapman \& Hall/CRC Data Mining and Knowledge Discovery Series.

Japkowicz, N. and Stephen, S. (2002). The class imbalance problem: A systematic study. Intell. Data Anal, 6(5):429-449.

Kotsiantis, S. B. (2009). Educational data mining: a case study for predicting dropoutprone students. IJKESDP, 1(2):101-111.

Macfadyen, L. and Dawson, S. (2010). Mining LMS data to develop an "early warning system" for educators: A proof of concept. Computers \& Education, 54(2):588-599.

Manhaes, L. M. B., da Cruz, S. M. S., Costa, R. J. M., Zavaleta, J., and Zimbrao, G. (2011). Previsão de estudantes com risco de evas ao utilizando técnicas de mineração de dados. Anais do XXII SBIE.

Rodrigues, R. L., de Medeiros, F. P. A., and Gomes, A. S. (2013). Modelo de regressão linear aplicador à previsã de desempenho de estudantes em ambiente de aprendizagem. Anais do XXIV SBIE.

Romero, C. and Ventura, S. (2010). Educational data mining: A review of the state of the art. IEEE Transactions on Systems, Man, and Cybernetics, Part C, 40(6):601-618. 